\title{
Getting less than their fair share: Maltreated youth are hyper-cooperative yet vulnerable to exploitation in a public goods game
}

\author{
Jan Keil ${ }^{1,2}$ (i) Sonja Perren ${ }^{3,4}$ (1) Andrea Schlesier-Michel ${ }^{1,5}$ \\ Fabio Sticca ${ }^{6}$ \\ Susan Sierau ${ }^{1,7}$ (i) \\ Annette M. Klein ${ }^{1}$ \\ Nikolaus Steinbeis ${ }^{8,9}$ \\ Kai von Klitzing ${ }^{1}$ \\ Lars O. White ${ }^{1}$
}

${ }^{1}$ Department of Child and Adolescent

Psychiatry, Psychotherapy and

Psychosomatics, University of Leipzig,

Leipzig, Germany

${ }^{2}$ Department of Social Monitoring and Methodology, German Youth Institute, Munich, Germany

${ }^{3}$ Department of Empirical Educational Research, University of Konstanz, Konstanz, Germany

${ }^{4}$ Thurgau University of Teacher Education, Kreuzlingen, Switzerland

${ }^{5}$ Department of Developmental

Psychology, Friedrich-Schiller-University of Jena, Jena, Germany

${ }^{6}$ Institute of Education, University of Zurich, Zurich, Switzerland

${ }^{7}$ Department for Medical Psychology and Medical Sociology, University of Leipzig,

Leipzig, Germany

${ }^{8}$ Department of Developmental

Psychology, Leiden University, Leiden, The Netherlands

${ }^{9}$ Department of Clinical, Educational and Health Psychology, University College London, London, UK

\section{Correspondence}

Jan Keil, Department of Child and Adolescent Psychiatry, Psychotherapy and Psychosomatics, University of Leipzig, Leipzig, Germany.

Email: jan.keil@medizin.uni-leipzig.de

Funding information

European Regional Development Fund;

Bundesministerium für Bildung und

Forschung, Grant/Award Number:

01KR1201A; German Federal Ministry

of Education and Research, Grant/Award

Number: 01KR1201A; LIFE-Leipzig

Research Center for Civilization Diseases,

University of Leipzig

\begin{abstract}
Human cooperative behavior has long been thought to decline under adversity. However, studies have primarily examined perceived patterns of cooperation, with little eye to actual cooperative behavior embedded within social interaction. Gametheoretical paradigms can help close this gap by unpacking subtle differences in how cooperation unfolds during initial encounters. This study is the first to use a childappropriate, virtual, public goods game to study actual cooperative behavior in 329 participants aged 9-16 years with histories of maltreatment $(n=99)$ and no maltreatment ( $n=230$ ) while controlling for psychiatric symptoms. Unlike work on perceived patterns of cooperation, we found that maltreated participants actually contribute more resources to a public good during peer interaction than their nonmaltreated counterparts. This effect was robust when controlling for psychiatric symptoms and peer problems as well as demographic variables. We conclude that maltreatment may engender a hyper-cooperative strategy to minimize the odds of hostility and preserve positive interaction during initial encounters. This, however, comes at the cost of potential exploitation by others.

KEYWORDS

cooperation, developmental psychopathology, game theory, maltreatment, public goods game
\end{abstract}


The human species is unique in terms of its skill and motivation to cooperate (Tomasello, 2014). At the same time, environmental factors are thought to figure prominently in shaping the specific level of cooperativeness of an individual (Belsky, Steinberg, \& Draper, 1991). As a core mechanism of environmental influence, repeated interactions with attachment figures are amalgamated over time into internal working models that become "increasingly a property of the child" (Bowlby, 1988, p. 127), guiding their behavior across social contexts (Cassidy, Jones, \& Shaver, 2013). Accordingly, adverse rearing conditions, such as maltreatment experiences, are thought to inculcate a less cooperative mindset toward others more generally, which may also serve as an adaptation to a stressful environment with high competition for resources (Belsky et al., 1991). In line with this, maltreatment gives rise to reports of decreased cooperativeness-often assessed via questionnaires on being helpful or sharing with peers-as well as reports of increased aggression and/or social withdrawal, which account for a wide range of peer problems, typically observed among maltreated children (Anthonysamy \& ZimmerGembeck, 2007; Bolger \& Patterson, 2001).

Simultaneously, an independent line of inquiry suggests that maltreated children may go out of their way to minimize the odds of hostile encounters with others. Scholars have thus empirically observed a set of behaviors involving compulsive compliance (Crittenden \& Ainsworth, 1989; Crittenden \& DiLalla, 1988) or indiscriminate friendliness/social disinhibition (Kay \& Green, 2013; Rutter et al., 2010) introducing theoretical concepts, such as costly altruism (Zahn-Waxler \& van Hulle, 2011) or excessive submissiveness (Sloman \& Taylor, 2016) following early adversity, including maltreatment. However, this research has yet to be reconciled with the aforementioned work showing diminished levels of cooperation in the wake of maltreatment.

In part, resolution of this inconsistency may have been hampered by limitations inherent in the methods of this field which primarily assess perceived cooperative behavior of children and adolescents, including self-, peer-, parent-, and teacher-rated behavior. While providing insight into broader patterns of social behavior, such methods are limited in their ability to account for the process whereby social exchanges initially unfold with unfamiliar interaction partners. In contrast, game-theoretical paradigms allow us to parse broad patterns of social exchanges into their constituent interactive units. They provide a rich set of powerful tools for quantifying interactive social behaviors mathematically (Gradin et al., 2016; Gummerum, Hanoch, \& Keller, 2008) and may therefore add a new and crucial dimension to prior work on social behavior in maltreated individuals.

\subsection{Cooperative behavior within social dilemmas}

Cooperative behavior has often been studied within gametheoretical paradigms (van Lange, Joireman, Parks, \& van Dijk, 2013). One particularly suitable class of game-theoretical paradigms to study developmental changes in cooperation in children

\section{RESEARCH HIGHLIGHTS}

- Though ample work shows that humans are uniquely cooperative, few studies examine how early adversity impinges on individual-level cooperative behavior, quantifiable via game-theoretical paradigms.

- Ours is the first study to use a public goods game to assess effects of maltreatment on actual cooperative behavior toward peers among 9- to 16-year-olds.

- We show that maltreated children actually contribute more resources than their nonmaltreated counterparts unlike previous work suggesting diminished cooperativeness.

- While this strategy may serve to minimize hostility during initial encounters, it also comes at the cost of potential exploitation by others.

and adolescents are social dilemmas (Crone, Will, Overgaauw, \& Güroğlu, 2014). Here, the individual gains most by pursuing a selfish strategy which conflicts with what would be best from a collective perspective (i.e., cooperation; Dawes, 1980). Due to its strategic makeup, the most stringent social dilemma to study cooperation in groups is the public goods game (PGG; Hardin, 1968), which is the multiperson case of a Prisoner's Dilemma (PD; Luce \& Raiffa, 1957; Rand \& Nowak, 2013). Public goods refer to resources consumable by everyone in the group irrespective of an individual's prior contribution (Olson, 1965), such as a clean environment or public services (Gummerum et al., 2008).

Despite ample research in adults (for overviews see Chaudhuri, 2011; Ledyard, 1995; Zelmer, 2003) much less is known about child and adolescent behavior in these situations. Recent work has begun to show that children from community samples initially contribute about the same amount of resources as adults and also display the tendency to cooperate conditionally (i.e., cooperate only when others cooperate; Harbaugh \& Krause, 2000; Keil et al., 2017; Vogelsang, Jensen, Kirschner, Tennie, \& Tomasello, 2014). Furthermore, older children as well as adolescents have been shown to more flexibly adjust their strategy as a function of their peer's behavior compared to younger children (Keil et al., 2017; van Hoorn, van Dijk, Meuwese, Rieffe, \& Crone, 2016).

Within the limited set of studies utilizing these paradigms in child and adolescent community samples, social dilemmas have recently also been introduced into developmental psychopathology. Some work in this area has implemented the trust game. In the trust game, a trustor can allocate resources to a trustee. Subsequently, these resources are augmented by a given factor and finally the trustee must decide how many resources to return to the trustor (Alarcón \& Forbes, 2017). ${ }^{1}$ Most importantly for the present purposes, a recent study using multiple trials of a singleshot trust game found that postinstitutionalized, adopted youth displayed lower rates and maintained less sharing behavior after they interacted with nonreciprocating peers, compared to neverinstitutionalized, nonadopted controls (Pitula, Wenner, Gunnar, \& 
Thomas, 2017). However, despite Pitula et al.'s (2017) intriguing results, it remains unclear whether their findings are generalizable to other more common forms of maltreatment or other types of social situations. Thus, institutionalization and/ or adoption may engender a distinct pattern of social behavior (e.g., involving limited social reciprocity) that is not typically linked with maltreatment without placement in foster care or adoption (see Zeanah \& Gleason, 2015). Furthermore, while a single-shot trust game accurately estimates a disposition to trust in a dyadic context (Alarcón \& Forbes, 2017), it cannot account for key aspects of more advanced group-level cooperation over multiple trials (e.g., higher motivation to cooperate; Axelrod, 1984; Blake, Rand, Tingley, \& Warneken, 2015). In the present research, we therefore implemented an iterative group-level PGG to capture effects of maltreatment on cooperative behavior across multiple interactions with the same coplayers.

Moreover, to assess the specificity of effects of maltreatment, it may be crucial to account for concurrent clinical symptoms. Notably, clinical symptoms are typical sequelae of maltreatment (Jaffee, 2017) and also coincide with less perceived cooperative behavior (Padilla-Walker, Carlo, \& Nielson, 2015). As far as clinical research on game-theoretical paradigms is concerned, scholars have primarily examined adult samples (Clark, Thorne, Hardy, \& Cropsey, 2013; King-Casas \& Chiu, 2012). Prior studies on adults with disruptive behavior symptoms consistently document attenuated levels of cooperative behavior compared to healthy controls using PDs (Mokros et al., 2008; Montañés, de Lucas, \& Rodríguez, 2003). For adults with internalizing problems (e.g., depression), studies have yielded mixed findings regarding their cooperative behavior in PGGs and PDs (for an overview see Alarcón \& Forbes, 2017). While some work reports higher rates of cooperation in depressed individuals (Sorgi \& van't Wout, 2016), other studies report lower rates of cooperation (Clark et al., 2013; Pulcu et al., 2015; Surbey, 2011) or no behavioral differences compared to healthy controls (Gradin et al., 2016).

Of the little work utilizing game-theoretical social dilemmas in child and adolescent samples, only few have investigated how psychiatric symptoms impact behavior in these situations (for reviews see Alarcón \& Forbes, 2017; Sharp, 2012). While research using the trust game demonstrates less trustworthiness among boys with externalizing problems (Sharp, Ha, \& Fonagy, 2011), children with more conduct symptoms display lower levels of, and are less likely to repair cooperation in a PD (Blake et al., 2015). Moreover, two studies report cooperative behavior among clinically referred adolescents compared to healthy controls in a PD. In the first study, anxiously depressed adolescents who played a PD with a computerized coplayer exhibited higher rates of cooperation following coplayer's cooperation, but no differences after their coplayer's defection (McClure et al., 2007). Conversely, in the second study using the same experimental setup, a sample of anxious adolescents evidenced higher rates of cooperative behavior following their coplayer's defection (McClure-Tone et al., 2011). Thus, while effects are clearly heterogeneous, clinical symptoms are a potential confounder when analyzing the impact of maltreatment on cooperative behavior. For this reason, we elected to control for clinical symptoms in the present study.

\subsection{The current study}

In this study we analyze cooperative behavior of maltreated and nonmaltreated children and adolescents within a computerized PGG called the Pizzagame (Keil et al., 2017). In the Pizzagame, participants are led to believe they are playing an online computer game with three sets of two same-aged, same-sex coplayers who are in fact computer generated and following preprogrammed scripts. The Pizzagame comprises three conditions. In the first condition, participants face cooperative coplayers, in the second condition selfish coplayers, and in the third condition coplayers with divergent strategies (i.e., cooperative and selfish). This approach first establishes baseline cooperative behavior and induces a large behavioral change by placing the exploitative strategy second (conditional cooperation). Additionally, the divergent condition taps into a potential recovery of cooperative behavior and the extent to which subjects side with a cooperative or an exploitative peer. We extrapolated absolute contributions in each condition and behavioral change between conditions.

This study pursues two main aims. First, we seek to analyze if and how maltreatment experiences impact cooperative behavior of children and adolescents in a PGG. According to the aforementioned literature, we tested whether maltreatment exposure gives rise either to (a) lower or, alternatively, (b) higher absolute contributions within each condition of our PGG. Moreover, we explored effects of maltreatment exposure on the flexibility to adapt contribution levels to behavioral shifts of coplayers between conditions. Second, we sought to establish the independent effect of maltreatment on cooperative behavior over and above the effect of concurrent psychopathology and peer problems. Both of these domains are well-known sequelae of maltreatment (Cicchetti \& Toth, 2016) and therefore establishing an independent effect of maltreatment on cooperation would underscore its fundamental importance for cooperation. Therefore, we tested the effects of maltreatment on cooperative behavior controlling for psychiatric symptoms and peer problems, both at the absolute level of cooperation and change between conditions.

\section{METHOD}

\section{$2.1 \quad$ Sample}

We recruited 408 participants aged 9-16 years from the community $(n=278)$, child psychiatric services $(n=104)$ and the child protection services (CPS; $n=26$ ) as part of an ongoing study analyzing the pathways from childhood maltreatment to psychiatric symptoms and disorders (for a detailed description see White et al., 2015). Ethical approval was obtained from the university's institutional review board (IRB). Informed consent and assent were obtained from caregivers and youth prior to participation. Following our established 
procedure to safeguard against limited task comprehension (Keil et al., 2017), we excluded 79 children and adolescents (46.1\% maltreated) because they erred on more than one of nine comprehension questions following instructions on the strategic makeup of the game. Excluded participants did not differ from the final sample population with regard to gender $(p=0.103, d=0.16)$, but regarding the proportion of maltreatment $(p=0.009, d=0.26)$ age $(p \leq 0.001$, $d=-0.66)$, monthly household income ( $p=0.012, d=0.26)$, and caregiver's school education ( $p \leq 0.001, d=0.51$ ). Given that our a priori inclusion threshold based on Keil et al. (2017) resulted in exclusion of a sizable number of participants, we conducted sensitivity analyses while relaxing the threshold (up to three errors in the comprehension questions), which only resulted in exclusion of $n=20$ participants (40\% maltreated). This sensitivity analysis involved a larger sample of $N=388$ children and adolescents who were comparable to the excluded children with regard to the proportion of maltreatment ( $p=0.406, d=0.01)$, gender $(p=0.169, d=0.14)$, as well as caregiver's monthly household income ( $p=0.106, d=0.16$ ), and merely differed from excluded children in terms of age ( $p \leq 0.001, d=-1.22)$ and caregiver's school education ( $p=0.001, d=0.81$ ). This model yielded comparable model fit and effects of maltreatment. Therefore, we decided to apply our a priori inclusion threshold (up to 1 error in the comprehension questions) for our main analysis, which yielded a final sample of $N=329$ participants. Tables $A 1$ and $A 2$ show demographic data as well as descriptive data on cooperative behavior in the PGG, psychopathology, peer problems, and maltreatment.

\section{$2.2 \quad$ Procedure}

Children and caregivers were invited to the laboratory for parallel assessments that lasted approximately $3 \mathrm{hr}$ and consisted of interviews, self-reports, and experimental set-ups. To ensure privacy, datacollection was carried out in separate rooms by trained researchers. Maltreatment interviews were coded after the appointment. Additionally, given prior permission, questionnaires were forwarded to the second caregiver and a teacher. After the appointment, the caregiver received a monetary reimbursement for participation while children and adolescents could choose a gift (see below).

\section{$2.3 \quad$ Instruments}

\subsubsection{Maltreatment}

The Maternal Maltreatment Classification Interview ( $\mathrm{MMCl}$; Cicchetti, Toth, \& Manly, 2003) was administered to caregivers and recorded for later coding. Lifetime presence of incidents involving one or more of six maltreatment subtypes was assessed using screening questions. Positive screens were followed up by assessing subtypes (sexual abuse, physical abuse, emotional maltreatment, failure to provide, lack of supervision, moral/legal/educational maltreatment), severity ( $1=$ low to $5=$ high), and developmental stages, that is, infancy (up to 1.4 years), toddlerhood (1.5-2 years), preschool age (3-5 years), early school age (6-7 years), late school age (8-12 years), and adolescence (13-18 years) per incident. Interviews were coded by trained researchers using the Maltreatment Classification System (MCS; Barnett, Manly, \& Cicchetti, 1993),which has proven valid and reliable (e.g., Manly, Oshri, Lynch, Herzog, \& Wortel, 2013). To ensure high data-quality, onsite training was provided by one of the authors of the MCS. If coding issues occurred, raters consulted a senior researcher board that met on a fortnightly basis and contacted the MCS trainer, if necessary. Three continuous variables (number of subtypes, chronicity, and maximum severity) were extracted from these measures. This approach was preferred given that continuous maltreatment measures do greater justice to the complexity of adverse experiences and are therefore superior to dichotomous measures of maltreatment (Manly, Kim, Rogosch, \& Cicchetti, 2001). To yield an age-independent index of maltreatment chronicity, we divided the number of developmental periods (e.g., toddlerhood, early childhood) in which participants had experienced maltreatment by the number of developmental periods they had already gone through (Sierau et al., 2017; White et al., 2017).

\subsubsection{Cooperative behavior in the PGG}

To assess cooperative behavior children played the Pizzagame (Keil et al., 2017), a novel, developmentally appropriate, computerized life-like task implementing the strategic setup of a PGG. Prior to starting the game, participants received thorough information regarding the rules and setup of the game (i.e., number of trials and players), followed by three example scenarios (i.e., cooperative, exploitative, noncooperative) illustrating potential outcomes of the game. Each of the three scenarios was followed by three comprehension questions to ensure that participants understood the strategic makeup of the PGG. Afterward, a test version of the game was run to familiarize participants with the game interface. To incentivize participants, they were told that the value of the gift they would receive at the end of the appointment would depend upon how many slices of pizza they retrieved in the course of the game. In reality, participants could all choose from the same set of presents to avoid disappointment due to differences in individual game behavior. Rather than a formal debriefing, children were exposed to an uplifting closing experience (i.e., the moderately cooperative condition followed by receiving a gift from the biggest box; see Thompson, 1990, pp. 11-12) in order not to jeopardize the trusting relationship to the experimenters for longitudinal assessments (for a complete description of the experimental setup see Keil et al., 2017).

At the start of the Pizzagame, participants were led to believe they were connected with other children over the Internet. In fact, participants played the Pizzagame with computer-generated coplayers with fixed strategies. Set at a virtual school, participants interacted with three pairs of same-age, same-sex peers. At the beginning of each round, players received an initial endowment of nine virtual slices of pizza. Without seeing other player's decisions, participants decided how many (0, 3, 6, or 9) slices they would like to take to school and pool to a "communal plate" (i.e., contribute to the public good) or leave at home (i.e., keep for themselves; Figure 1a). 
(a)

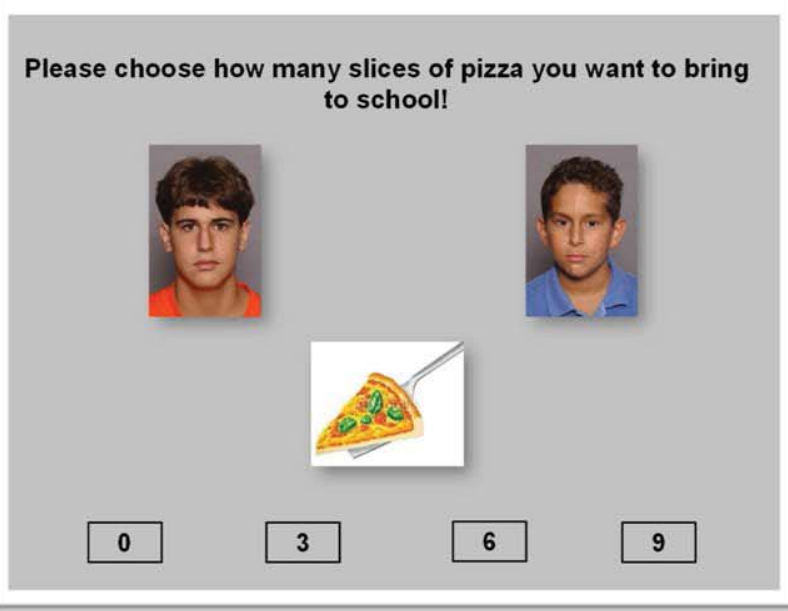

(c)

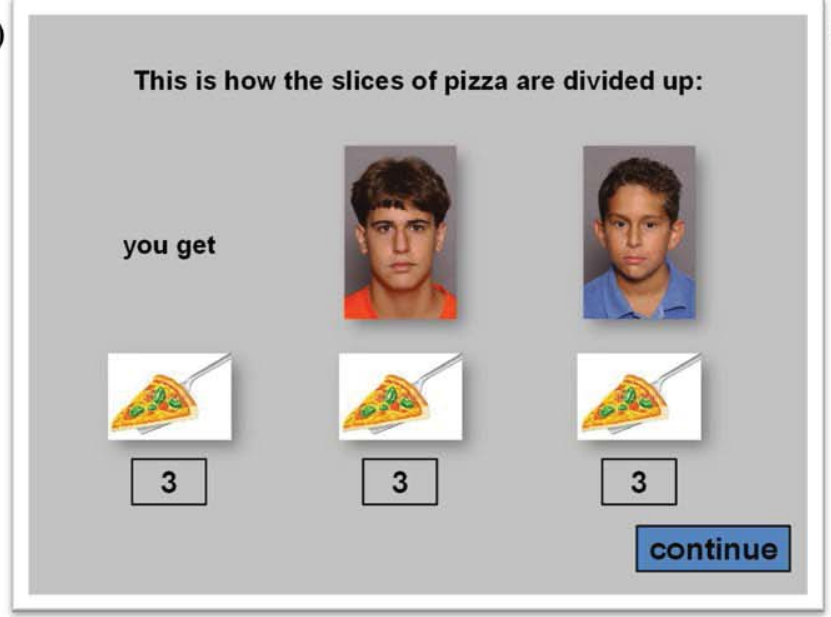

(b)

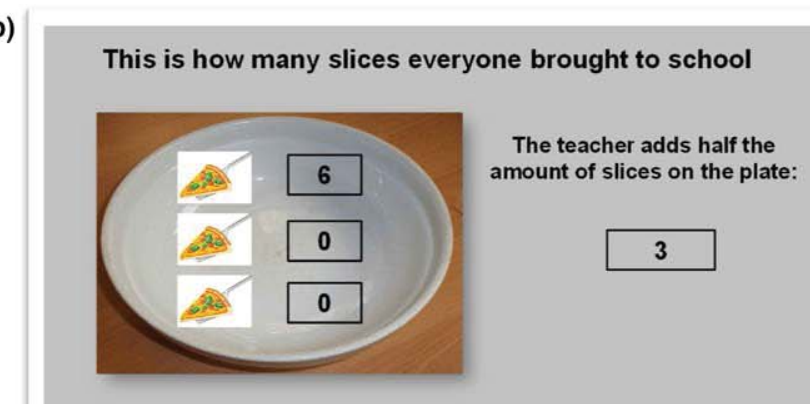

Together, you have gathered this many slices of pizza in this round:

9 (d)

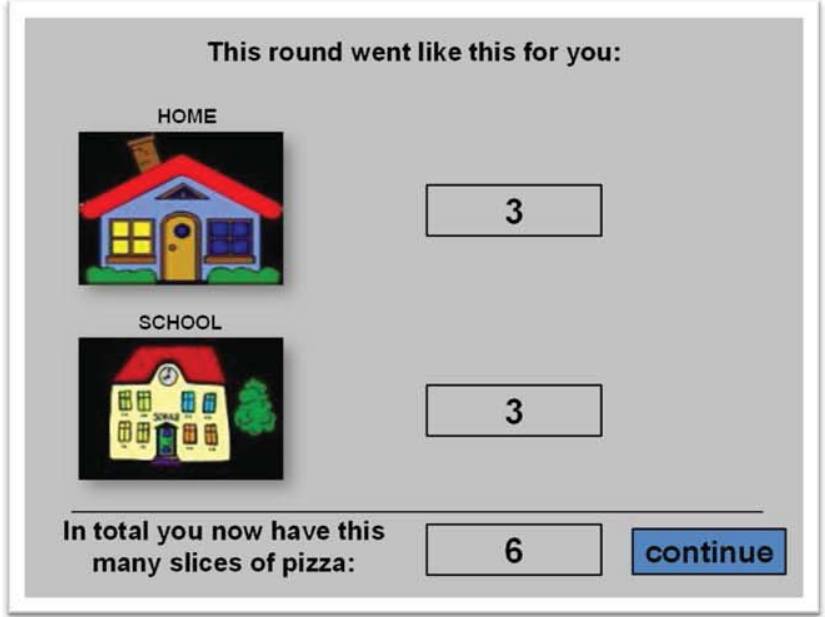

FIGURE 1 Illustration of four key stages of a hypothetical round of the Pizzagame: (a) Decision situation with photographs of coplayers and choice of contributions. (b) Presentation of participants' contribution (here six) along with anonymous individual contributions of coplayers and augmentation by teacher (50\% of the sum of individual contributions). (c) Illustration of the redistribution of the public good to each individual player. (d) Illustration of the surplus of resources after one round of the Pizzagame. Photographs in the task and figure are drawn from the NIMH Child Emotional Faces Picture Set (NIMH-ChEFS; Egger et al., 2011)

At school, the virtual teacher augmented what was on the communal plate by adding $50 \%$ of the sum of all pooled slices of pizza (Figure 1b). All slices on the communal plate were then redistributed equally among players irrespective of each player's initial contribution (Figure 1c). At the end of each round, slices obtained at school and those left at home were summed for each player, representing each individual's outcome for the respective round (Figure 1d). The Pizzagame progressed through three conditions comprising four rounds each. In the first condition, participants faced highly cooperative coplayers who both contributed all of their initial endowment to the public good in the first round. In the following three rounds, one coplayer carried on contributing nine slices, while the other coplayer slightly reduced contributions to six slices of pizza. In the second condition, coplayers pursued a selfish strategy by contributing three and zero slices of their initial endowment to the public good and reducing contributions to the minimum amount (both zero slices) in the subsequent three rounds. In the third condition, coplayers displayed a divergent strategy with one coplayer contributing all of his initial resources while the other coplayer contributed three slices in the first round and none in the following three rounds.

\subsubsection{Psychiatric symptoms and peer problems}

To assess psychiatric symptoms the Strengths and Difficulties Questionnaire (SDQ; Goodman, 1997) was administered to primary caregivers (two, if possible), children, and teachers. The SDQ comprises 25 items (three-point scale) yielding five symptom scales (conduct problems, hyperactivity, emotional symptoms, peer problems, and prosocial behavior). For this study, the subscales emotional symptoms, conduct problems, hyperactivity, and peer problems were used from up to four informants. The reports on psychiatric symptoms and peer problems by the different informants yielded acceptable internal consistency (emotional problems, $\alpha=0.76$; conduct problems, $\alpha=0.77$; hyperactivity, $\alpha=0.80$, peer problems, $\alpha=0.78$ ). 


\subsection{Data analyses}

To test our hypotheses we applied structural equation modeling (SEM) using Mplus 7.11 (Muthén \& Muthén, 2013). To model maltreatment as a continuous latent variable we used a formative approach (Diamantopoulos \& Winklhofer, 2001). Unlike a reflective approach, a formative approach assumes that indicators cause the latent variable instead of vice versa (MacCallum \& Browne, 1993). More specifically, maltreatment is more appropriately conceptualized as composite index (formative) because the different indicators (dimensions) are not interchangeable and, crucially, high scores on one dimension of the maltreatment experiences do not necessarily imply high scores on other dimensions.

To model cooperative behavior, we specified a latent reflective variable for each of the three conditions of the Pizzagame (i.e., cooperative, selfish, divergent) not including the first round of each condition. In so doing, we sought to ensure that participants had been cued regarding the behavioral disposition of their coplayers and could factor this information into their decision. This measure also aimed to reduce potential carryover effects between conditions that might have biased latent means (Keil et al., 2017).

To model psychiatric symptoms and peer problems, we applied a multisource approach (Kraemer et al., 2003) combining information from caregivers, children, and teachers to specify latent reflective variables representing the level of emotional symptoms, hyperactivity, conduct problems, and peer problems. No cross loadings were specified. For the latent variables reflecting cooperative behavior, psychopathology, and peer problems we specified autocorrelated residuals (Sörbom, 1975) between the corresponding observed indicators, both for conditions (i.e., the respective number of round within the different conditions) and the psychiatric symptoms and peer problem subscales (i.e., items of the respective respondents). The effect coding method (Little, 2013) was used for the identification of latent mean scores regarding the cooperative behavior in each condition and the psychiatric symptom subscales. Age, gender, caregiver's school education, and household net income were used as manifest control variables.

The analyses were carried out in three steps. First, we conducted bivariate analyses to examine the links among cooperative behavior, maltreatment, psychopathology, and peer problems without controlling for one another. Second, we specified a latent state model (LSM) regressing each condition of the Pizzagame on the maltreatment composite as well as control variables. To test the influence of maltreatment on behavioral flexibility (change in contributions between conditions) we expanded the LSM to an autoregressive model (ARM) by additionally specifying autoregressive paths from the cooperative to the selfish and from the selfish to the divergent condition. Third, to check whether a possible impact of maltreatment on cooperative behavior was robust after controlling for psychopathology and peer problems, we included the three psychiatric symptoms subscales and the peer problem subscale into the LSM of Step 2. Specifically, we regressed each condition of the Pizzagame on the maltreatment composite, the three psychiatric symptoms subscales, the peer problem subscale, and the control variables, as well as the psychiatric symptoms subscales and the peer problem subscale on the maltreatment composite and the control variables. To check if a possible influence of maltreatment experiences on behavioral flexibility remained significant after controlling for psychiatric symptoms and peer problems, we again expanded the LSM to an ARM. Here, we specified autoregressive paths from the cooperative to the selfish and from the selfish to the divergent condition.

To evaluate the model fits of the LSMs and ARMs we used (a) the chi-square statistic, (b) the comparative fit index (CFI), (c) the root-mean-squared error of approximation (RMSEA), and (d) the standardized root-mean-squared residual (SRMR). Following $\mathrm{Hu}$ and Bentler (1999), a RMSEA $\leq 0.05$ (0.08), a CFI $\geq 0.95$ (0.90), and a SRMR $\leq 0.05$ (0.08) indicate a good (adequate) model fit.

\section{RESULTS}

The bivariate analyses of Step 1 indicated that maltreated children contributed more resources in every round of every condition than nonmaltreated children (Figure 2). Moreover, only the number of maltreatment subtypes, maltreatment severity, and the peer problems subscale were associated with higher levels of cooperation toward cooperative coplayers. Besides this, the full set of psychiatric symptom variables, peer problems and maltreatment dimensionsexcept maltreatment chronicity and hyperactivity in the divergent condition-were related to higher levels of cooperation toward selfish and divergent coplayers. In addition, all three maltreatment dimensions were positively associated with all psychiatric symptom subscales and the peer problem subscale (Table A3).

The first LSM of Step 2 showed an adequate model fit. It revealed that children and adolescents with more maltreatment exposure were more cooperative toward cooperative, selfish, and divergent coplayers. $^{2}$ The ARM of Step 2 testing behavioral change between conditions also showed an adequate model fit. It indicated that maltreatment exposure showed no significant effect on changes in contributions from the cooperative to the selfish or from the selfish to the divergent condition (Table A4).

The LSM of Step 3 also revealed an adequate model fit. The bivariate associations between cooperative behavior and psychiatric symptoms depicted in Step 1 were abolished after controlling for the effect of maltreatment on psychiatric symptoms with the exception of the positive effect of emotional symptoms on cooperation in the divergent condition. In contrast, the bivariate associations between cooperative behavior in the cooperative and the divergent condition and peer problems depicted in Step 1 remained robust to controlling for the effect of maltreatment on peer problems. Moreover, as predicted, results indicated that youth with more exposure to maltreatment experiences were more cooperative toward cooperative, selfish, and divergent coplayers after controlling for psychiatric symptoms and peer problems. Furthermore, youth with more exposure to maltreatment displayed higher levels of emotional 


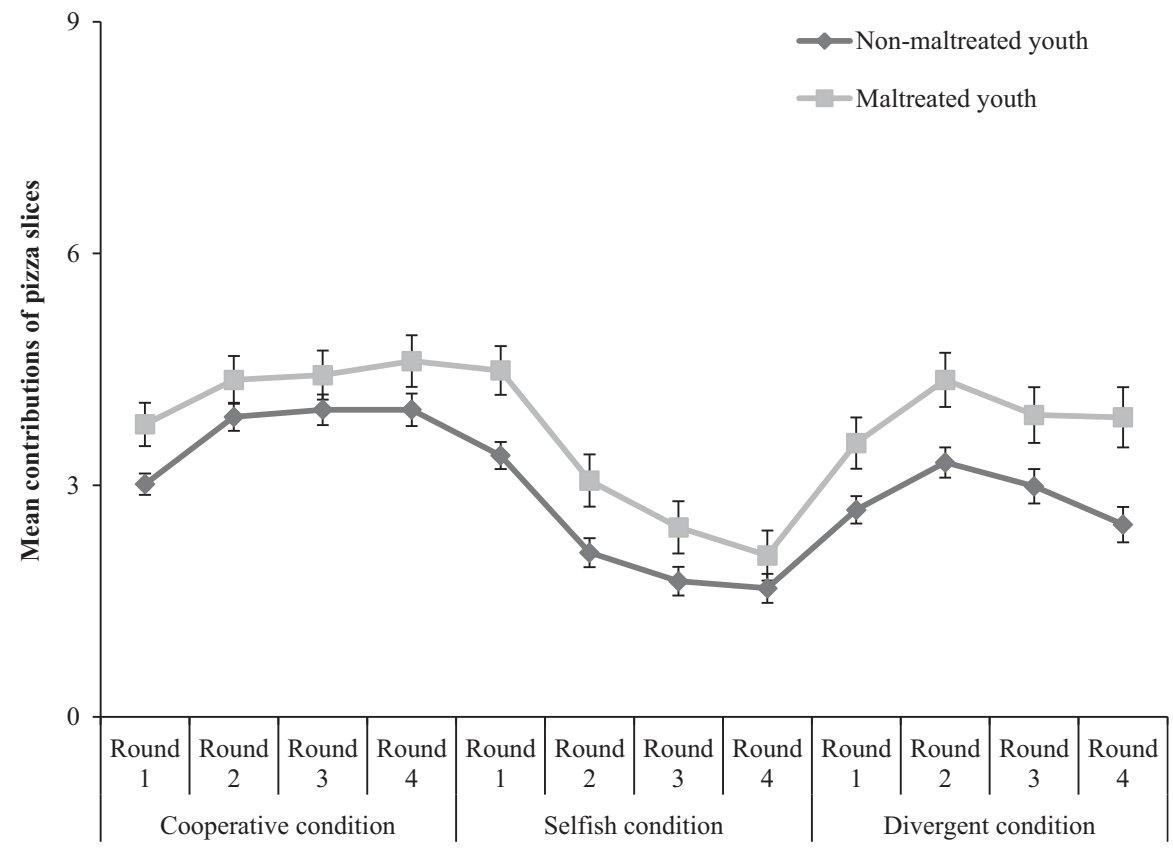

FIGURE 2 Mean contributions with standard errors for the cooperative, selfish, and divergent condition for maltreated $(n=99)$ and nonmaltreated $(n=230)$ children

Rounds of the three conditions of the Pizzagame

symptoms, conduct problems, and hyperactivity (Figure 3 and Table A4). The ARM of Step 3 testing behavioral change between conditions showed an adequate model fit. It indicated that maltreatment exposure showed no effect on changes in contributions from the cooperative to the selfish and from the selfish to the divergent condition (Table A4).

The following additional results emerged: Females contributed more toward cooperative coplayers prior to controlling for psychiatric symptoms than males (LSM Step 2). Before and after controlling for psychiatric symptoms and peer problems (LSM
Step 2, LSM Step 3), older children contributed less toward selfish coplayers and children with less educated parents contributed more toward selfish coplayers. Older children evidenced a more pronounced decrease in contributions from cooperative to selfish, and a more pronounced increase of contributions from selfish to divergent coplayers (ARM Step 3). With increasing education of caregivers, children showed a more pronounced decrease in contributions from the cooperative to the selfish condition (ARM Step 3). Additionally, girls showed higher levels of internalizing problems while boys displayed more externalizing and peer problems.

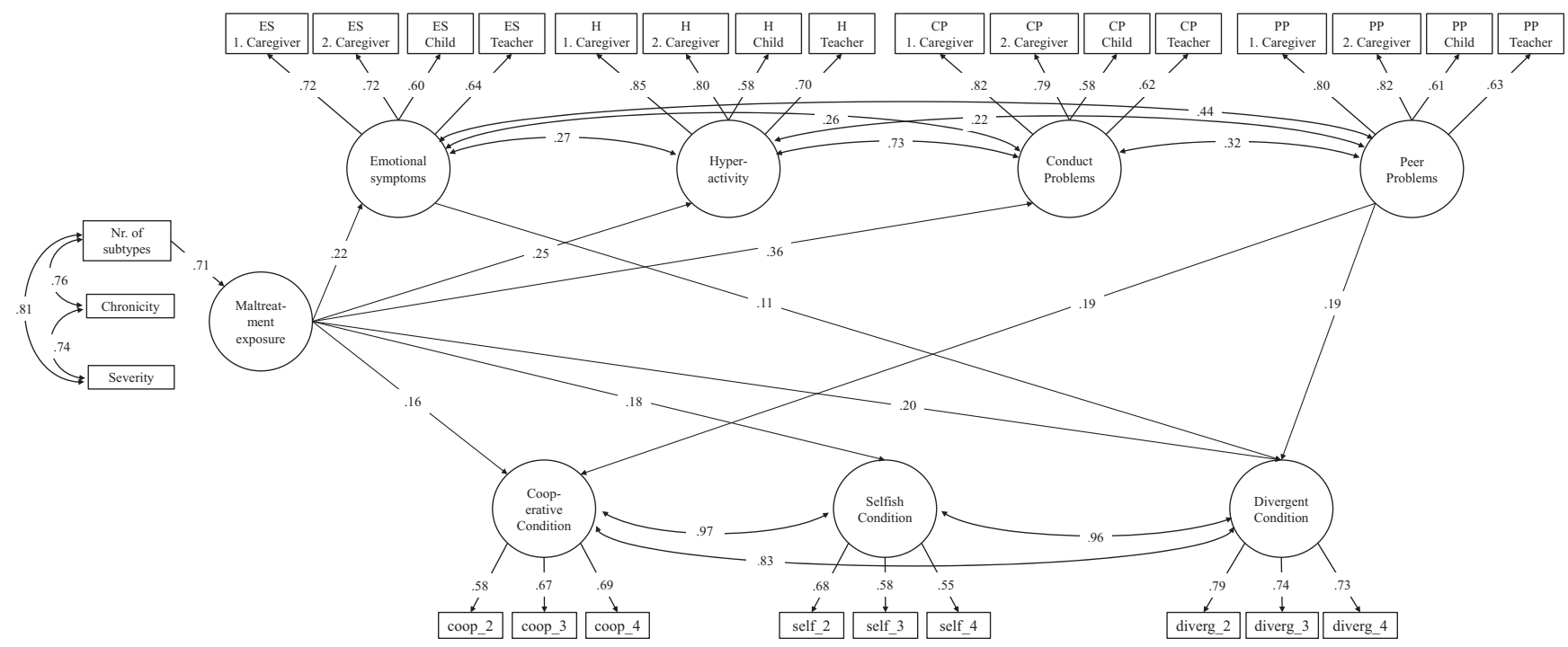

FIGURE 3 Latent state model of step 3 with standardized coefficients and paths between the three latent variables of the corresponding conditions (cooperative, selfish, and divergent conditions), the maltreatment composite controlling for psychiatric symptoms, age, gender, caregiver education, and monthly household income. Only statistically significant paths are displayed. For reasons of legibility, associations between latent constructs and manifest control variables (age, gender, income, education) are not displayed 
Finally, higher levels of externalizing and peer problems coincided with lower monthly household income while higher levels of externalizing problems were also associated with caregiver education (Table A4).

\section{DISCUSSION}

Ours was the first study aiming to investigate the impact of maltreatment experiences on actual and objectively assessed cooperative behavior of children and adolescents in a newly developed, computerized PGG. Moreover, given the well-established association between maltreatment and psychopathology, we also sought to analyze these effects while controlling for psychiatric symptoms and peer problems. We found that maltreated children exhibited increased levels of cooperative behavior, regardless of whether they played with cooperative, selfish, or mixed cooperative-selfish age-mates.

Notably, a similar pattern of increased cooperativeness emerged for emotional symptoms, conduct problems, hyperactivity, and peer problems in the bivariate analyses, raising the question whether the effects are primarily an emergent property of psychopathology and/ or peer problems rather than maltreatment. However, additional analyses adjusting for levels of psychopathology, peer problems, and relevant confounders (age, gender, SES), demonstrated that the effect of maltreatment was robust across all conditions. This intriguing pattern of results leads us to conclude that-far from invariably acting antisocially and uncooperatively-exposure to maltreatment may lead children and adolescents to act in an oversacrificing or hypercooperative manner toward cooperative as well as exploitative peers during the first few interactions.

On the face of it, our findings of maltreatment-related hypercooperativeness appear to challenge the view that maltreatment promotes antisocial behavior. However, in our data, maltreatment still coincided with increased antisocial behaviors, as indexed by a multi-informant measure of conduct problems, thus replicating the relationship typically reported in studies using questionnaire measures of social behavior (e.g., Anthonysamy \& Zimmer-Gembeck, 2007; Kim \& Cicchetti, 2010). At the same time, our bivariate analyses revealed that conduct problems were also associated with increased cooperativeness in our PGG when at least one of the coplayers acted selfishly. So, how is it possible that maltreatment simultaneously increases the odds for both antisocial problems as well as cooperative behavior? Potentially, this apparent contradiction may be attributable to the difference between measures of perceived interpersonal behavior and game-theoretical tasks capturing actual interpersonal behavior. For the former, informants evaluate actions of a specific child, potentially leading to recall of the most salient situations where the child displayed particularly abnormal social behaviors. By contrast, our PGG unpacks subtle differences in how cooperation unfolds during an initial encounter with unfamiliar peers. This situation could thus reflect participants' initial cooperative motivation when they get to know people, indicating that maltreated youth are more cooperative when entering a new group.

A hyper-cooperative strategy may make a great deal of sense assuming that maltreated youth would initially follow the goal of reducing the odds of hostile and/or increasing the odds of benign encounters with others (Sloman \& Taylor, 2016). However, this type of submissive behavior may make them easy targets for exploitation by others-as was particularly evident when they interacted with selfish coplayers in our PGG. It is possible that following a lengthier exploitative interaction than the one in the Pizzagame, maltreated participants would have eventually reverted to hypo-cooperation and disruptive behavior. Indeed, such a shift from hyper- to hypocooperation would reconcile our findings somewhat with prior work showing that youth with higher conduct problems display less reparation following disruption of cooperative interactions in a PD (Blake et al., 2015). However, these speculations remain tentative based on the current data, as we detected no evidence of hypo-cooperation in our PGG. Moreover, the clinical literature shows that disruptive behavior may actually co-occur with indiscriminate friendliness toward strangers following severe deprivation (Zeanah \& Gleason, 2015)-in other words, cooperative and disruptive behaviors are by no means mutually exclusive, but may instead vary as a function of context (e.g., familiarity of the target).

Besides this, it is also intriguing that our results markedly contrast with Pitula et al.'s (2017) findings in postinstitutionalized adoptees who showed lower rates and maintenance of sharing with peers in a trust game. One possibility is that institutionalization engenders a distinct form of social deficit, for example, involving diminished levels of reciprocity (Zeanah \& Gleason, 2015) as compared to maltreatment by caregivers encountered in the community. Yet, in comparing the findings of our study to Pitula et al.'s (2017), it is crucial to also bear in mind the difference between the dyadic and sequential nature of the single-shot trust game they used relative to the group-level, nonsequential, but iterative nature of our PGG. Thus, repeatedly interacting with the same peers across multiple trials, as was the case in our PGG, may create a stronger motivation to cooperate ("shadow of the future"; Axelrod, 1984; Blake et al., 2015; van Lange, Klapwijk, \& van Munster, 2011). By contrast, a single-shot trust game may inhibit such processes and better capture an initial disposition to trust.

Additional mechanisms may also apply, given the high levels of emotional maltreatment (e.g., excessive criticism, witnessing parental violence) in our sample which may be related to parental distress (Belsky \& Jaffee, 2006). Zahn-Waxler and van Hulle (2011) suggested that distressed caregivers may elicit excessive feelings of guilt, anxiety, and sadness in their offspring, ultimately prompting them to engage in costly altruism, designed to compensate feelings of guilt. Indeed, evidence for the importance of guilt for the present purposes is also highlighted by recent game-theoretical research. This work identifies guilt aversion-the avoidance of guilt that others will get less than they expect-as an important source of increased contributions in PGGs (Dufwenberg, Gächter, \& Hennig-Schmidt, 2011).

Notably, an alternative explanation also demands attention. Hence, it might be suggested that maltreated children were generally 
less engaged in the Pizzagame than nonmaltreated children, thus more liberally relinquishing resources irrespective of the behavior of their coplayers. However, maltreated and nonmaltreated participants showed comparable tendencies to adapt to behavioral shifts of their coplayers, indicating a similar level of attention and responsiveness to coplayer's behaviors. Indeed, Crittenden and DiLalla (1988) show that maltreated children retain high levels of flexibility in their compliant behavior, acting less compliantly when partnered with less controlling adults. The authors argue that this result reflects a continuing sensitivity to environmental changes in these children which is adaptive in adverse rearing conditions to ensure the best possible developmental outcome. Pitula et al.'s (2017) data on post- and noninstitutionalized children, who more flexibly adapted their behavior toward peers in a trust game suggesting a heightened sensitivity to reciprocation and defection, may also offer a case in point.

Beyond our main results, our data reveal that children with emotional problems contributed more when only one coplayer acted selfishly (divergent condition) after accounting for effects of maltreatment. This finding is partially consistent with the pattern of increased cooperativeness among adolescents with internalizing symptoms using the prisoners' dilemma (McClure et al., 2007; McClure-Tone et al., 2011). On a theoretical level, it conforms to the mechanism described by Zahn-Waxler and van Hulle (2011) that assumes costly (pathological) altruism to characterize internalizing problems. McClure et al. (2007) suggest that this may reflect a sociotropic interpersonal style, rendering children reluctant to take interpersonal risks inherent in many social relationships in order to maintain positive interactions at high costs. Compellingly, other work in this field suggests that sociotropy is associated with interpersonal guilt and self-blame (Robins, Bagby, Rector, Lynch, \& Kennedy, 1997), which may explain tendencies for excessive altruism following adversity. However, it is not clear why children with internalizing problems in the present study demonstrate more cooperative behavior only in the last divergent condition after taking maltreatment into account.

Notably, maltreatment also predicted the level of cooperation over and above the effects of peer problems. This is particularly intriguing, given that the Pizzagame simulated a peer interaction. From this pattern we therefore conclude that family-level maltreatment may give rise to internal representations that guide cooperative behavior in new social encounters (Bowlby, 1988) and these effects are not simply attributable to concurrent peer problems that may emerge in the wake of maltreatment (Cicchetti \& Toth, 2016). Nevertheless, peer problems appear to additionally give rise to increased cooperative behavior under cooperative and divergent conditions, independent of maltreatment, suggesting that peer problems may also be an important contributor to cooperative behavior toward peers. One possibility is that hyper-cooperativeness may potentially be part of a mechanism whereby children insufficiently defend themselves, making them "easy targets" for victimization by peers (e.g., Olweus, 2001).

Furthermore, the results mesh well with previous community sample findings (Keil et al., 2017), that is, older children more flexibly adapt their behavior to that of their coplayers, such that they exhibit greater tendencies to both decrease their cooperation with uncooperative peers as well as increase their cooperative behavior again with divergent peers (conditional cooperation). This age pattern has now also proven robust in the context of maltreatment experiences, psychiatric symptoms, and peer problems in this study. Our robust age effect therefore provides a way of reconciling discrepancies in the literature on age effects in PGGs (e.g., Cipriani, Giuliano, \& Jeanne, 2013; Fan, 2000; Sally \& Hill, 2006), by suggesting that older children neither cooperate more or less than younger children, but rather cooperate more conditionally, that is, adapting more flexibly to the strategies of their coplayers. Moreover, the fact that neither maltreatment nor psychiatric symptoms and peer problems affected the change in contributions between games suggests that these factors impact cooperative behavior at a different level (i.e., absolute contributions in each game) compared to age.

Finally, the the overall pattern that maltreatment experiences and peer problems better account for variance in cooperative behavior than psychiatric symptoms emphasizes the importance of considering these constructs in a single model. This finding should alert future scholarship to the possibility that effects of psychiatric symptoms may at least partly be attributable to exposure to adversity (Teicher \& Samson, 2013). Moreover, our replication of the well-established links between maltreatment and psychopathology as well as between gender and psychopathology lends further credence to our results, suggesting that they may also generalize to other samples. Of note, our assessment of psychiatric symptoms and peer problems included child self-reports alongside teacher and parent reports. While the sole use of self-reports to assess psychiatric symptoms has been critiqued, in their landmark article, Kraemer et al. (2003) surmise that each source contributes valuable information regarding the psychiatric symptoms of a person. The multi-informant approach used in this study (see Kraemer et al., 2003)-factor analytically pooling reports of children, teachers, and parents-accounts for measurement error and estimates the latent construct of psychiatric symptoms and peer problems independent of reporter perspective, thus representing a methodological advance compared to prior research in this area.

Some limitations deserve mentioning. First, the analyses of the current study are based on cross-sectional data indicating that the direction of effects should be interpreted cautiously. However, while the data on maltreatment experiences were collected retrospectively, cooperative behavior was assessed on-line in the laboratory, yielding a temporal order consistent with causality. Second, our PGG progresses through three consecutive conditions that are always presented in a fixed order (i.e., cooperative, selfish, and mixed cooperative-selfish round) and are thus not counterbalanced to control for any order effects. However, we opted for this fixed sequence for several reasons. One reason lies in the stratification demands this step would have placed on our sample, another in concerns about starting the paradigm with a condition that might diminish participants' engagement, such as the uncooperative or the divergent conditions (see Keil et al., 2017 for details). Third, our study did not distinguish between different subtypes of adversity and maltreatment as this would have placed much greater demands on our 
study in terms of sample size. In the literature on early adversity and maltreatment, there is an ongoing debate as to the specificity with which different subtypes (e.g., deprivation vs. threat) may give rise to distinct developmental pathways or not (Evans, Li, \& Whipple, 2013; McLaughlin, Sheridan, \& Lambert, 2014; Vachon, Krueger, Rogosch, $\&$ Cicchetti, 2015). This work provides a potentially important hint regarding a possible reason for the differences between our study's pattern of hyper-cooperativeness among youth with a wide range of maltreatment experiences and the diminished trust reported by Pitula et al. (2017) in a postinstitutionalized cohort who presumably primarily experienced severe deprivation and early separation.

In sum, our work clearly underscores the unique potential of game-theoretical paradigms for informing theories of human cooperation. Moreover, our main result that maltreatment exposure gives rise to elevated levels of youth's actual cooperative behavior over and above the effect of mental health as well as peer problems and controlling for age, gender, and SES raises a number of important points. First, the extent to which hyper-cooperativeness toward unfamiliar peers generalizes to other contexts, including the family and longer term relationships as well as its real-life correlates within interactions, will be an important avenue for future research. Overcompliance toward caregivers (Crittenden \& DiLalla, 1988), excessive submissiveness (Sloman \& Taylor, 2016), and indiscriminate friendliness toward strangers (Kay \& Green, 2013) have been observed among maltreated versus nonmaltreated individuals and are candidate behaviors that may partly correspond to hyper-cooperativeness we observed here. Second, future research should consider the psychobiological mechanisms underpinning hyper-cooperative behavior among maltreated youth. To this end, altered stress-hormone secretion may be a viable candidate, given its important role as a mediator of effects of maltreatment experiences on perceived antisocial (e.g., White et al., 2017) and prosocial behavior (e.g., Alink, Cicchetti, Kim, \& Rogosch, 2012). Third, our data suggest that youth are at risk of becoming exploited by their peers, which might inform future interventions aiming to buffer the adverse effects of maltreatment in childhood. Beyond this, our findings highlight the importance of considering differences in adverse social experience when conducting research into social deficits associated with psychopathology. Finally, our work indicates that game-theoretical paradigms offer much promise of quantifying subtle differences in social behavior not always obvious to the naked eye. Thereby, these paradigms bear the unique potential of vastly extending psychological and psychiatric research, which, to date, has been steeped almost exclusively in a tradition of assessing broad patterns of perceived social behavior reported by various informants.

\section{ACKNOWLEDGEMENTS}

The preparation of this article and the research presented herein were supported by grants from the German Federal Ministry of Education and Research (grants 01KR1201A to E) as well as by LIFE-Leipzig Research Center for Civilization Diseases, University of Leipzig. LIFE is funded by means of the European Union, by the European Regional Development Fund (ERDF) and by means of the Free State of Saxony within the framework of the excellence initiative. The responsibility for the contents of this publication lies with the authors.

\section{CONFLICT OF INTEREST STATEMENT}

The authors declare that the research was conducted in the absence of any commercial or financial relationships that could be construed as a potential conflict of interest.

\section{ENDNOTES}

${ }^{1}$ Similar to the PGG (described below), players gain the highest payoff in the long run (i.e., in an iterated version of the game) by cooperating (i.e., choosing to trust or exhibiting trustworthiness), but in the short run the most profitable decision is to defect to minimize risk (i.e., choosing not to trust) and obtain the highest payoff by not revealing trustworthiness (Crone et al., 2014). However, unlike the PGG, the trust game typically involves (a) a dyad, not a group, (b) a setup whereby players adopt inherently asymmetrical roles (trustor and trustee), and (c) sequential contributions, whereby one player directly responds to the other player's initial offer. Importantly, these features of the trust game mean that any rupture of trust (low initial contribution or return) is directly traceable to the specific player. Conversely, in the event of a players' defection in the PGG the defector may not necessarily be readily identifiable if contributions were made anonymously, thus facilitating freeriding behaviors.

${ }^{2}$ As an additional step, we also specified an alternative LSM with categorical maltreatment status (maltreated or nonmaltreated) as a manifest dichotomous predictor variable. This yielded comparable results to the LSM with a composite variable of maltreatment dimensions, showing that maltreated youth contributed more in the selfish and the divergent conditions than their nonmaltreated counterparts. However, we opted for a dimensional approach to do justice to the complexity of maltreatment given that it retains the variance within the maltreated group (increased sensitivity) which was also reflected in a slightly better model fit.

\section{ORCID}

Jan Keil (iD https://orcid.org/0000-0002-5001-0659

Sonja Perren (iD) https://orcid.org/0000-0002-7916-4037

Andrea Schlesier-Michel (iD https://orcid.org/0000-0003-3137-4438

Fabio Sticca (iD) https://orcid.org/0000-0003-3246-5833

Susan Sierau (iD) https://orcid.org/0000-0002-9849-6141

Annette M. Klein iD https://orcid.org/0000-0001-8246-4666

Nikolaus Steinbeis (iD) https://orcid.org/0000-0001-8650-4725

Kai Klitzing (iD https://orcid.org/0000-0003-3010-5121

Lars O. White iD https://orcid.org/0000-0001-7185-9929

\section{REFERENCES}

Alarcón, G., \& Forbes, E. E. (2017). Prosocial behavior and depression: a case for developmental gender differences. Current Behavioral 
Neuroscience Reports, 39(8), 117-127. https://doi.org/10.1007/ s40473-017-0113-x

Alink, L. R. A., Cicchetti, D., Kim, J., \& Rogosch, F. A. (2012). Longitudinal associations among child maltreatment, social functioning, and cortisol regulation. Developmental Psychology, 48(1), 224-236. https://doi. org/10.1037/a0024892

Anthonysamy, A., \& Zimmer-Gembeck, M. J. (2007). Peer status and behaviors of maltreated children and their classmates in the early years of school. Child Abuse \& Neglect, 31(9), 971-991. https://doi. org/10.1016/j.chiabu.2007.04.004

Axelrod, R. M. (1984). The evolution of cooperation. New York, NY: Basic Books.

Barnett, D., Manly, J. T., \& Cicchetti, D. (1993). Defining child maltreatment: The interface between policy and research. In D. Cicchetti \& S. L. Toth (Eds.), Child abuse, child development, and social policy (Vol. 8, pp. 7-73). New York, NY: Ablex.

Belsky, J., \& Jaffee, S. R. (2006). The multiple determinants of parenting. In D. Cicchetti \& D. J. Cohen (Eds.), Theory and method: Vol. 2. Developmental psychopathology (2nd ed., Vol. 3, pp. 38-85). New York, NY: John Wiley \& Sons.

Belsky, J., Steinberg, L., \& Draper, P. (1991). Childhood experience, interpersonal development, and reproductive strategy: An evolutionary theory of socialization. Child Development, 62(4), 647-670. https:// doi.org/10.2307/1131166

Blake, P. R., Rand, D. G., Tingley, D., \& Warneken, F. (2015). The shadow of the future promotes cooperation in a repeated prisoner's dilemma for children. Scientific Reports, 5(14559), 1-9.

Bolger, K. E., \& Patterson, C. J. (2001). Developmental pathways from child maltreatment to peer rejection. Child Development, 72(2), 549568. https://doi.org/10.1111/1467-8624.00296

Bollen, K., \& Lennox, R. (1991). Conventional wisdom on measurement: A structural equation perspective. Psychological Bulletin, 110(2), 305314. https://doi.org/10.1037/0033-2909.110.2.305

Bowlby, J. (1988). A secure base: Parent-child attachment and healthy human development. New York, NY: Basic Books.

Cassidy, J., Jones, J. D., \& Shaver, P. R. (2013). Contributions of attachment theory and research: A framework for future research, translation, and policy. Development and Psychopathology, 25(4), 1415-1434. https://doi.org/10.1017/S0954579413000692

Chaudhuri, A. (2011). Sustaining cooperation in laboratory public goods experiments: A selective survey of the literature. Experimental Economics, 14(1), 47-83. https://doi.org/10.1007/ s10683-010-9257-1

Cicchetti, D., \& Toth, S. L. (2016). Child maltreatment and developmental psychopathology: A multilevel perspective. In D. Cicchetti (Ed.), Maladaption and psychopathology: Vol. 3. Developmental psychopathology (3rd ed., pp. 457-512). New York, NY: Wiley. https://doi. org/10.1002/9781119125556

Cicchetti, D., Toth, S. L., \& Manly, J. T. (2003). Maternal maltreatment interview. Unpublished Manuscript, University of Rochester, Mt. Hope Family Center, Rochester, NY.

Cipriani, M., Giuliano, P., \& Jeanne, O. (2013). Like mother like son? Experimental evidence on the transmission of values from parents to children. Journal of Economic Behavior \& Organization, 90, 100-111. https://doi.org/10.1016/j.jebo.2013.03.002

Clark, C. B., Thorne, C. B., Hardy, S., \& Cropsey, K. L. (2013). Cooperation and depressive symptoms. Journal of Affective Disorders, 150(3), 1184-1187. https://doi.org/10.1016/j.jad.2013.05.011

Crittenden, P. M., \& Ainsworth, M. D. S. (1989). Child maltreatment and attachment theory. In D. Cicchetti \& V. Carlson (Eds.), Child maltreatment. Theory and research on the causes and consequences of child abuse and neglect (pp. 432-463). Cambridge, UK: Cambridge University Press. https://doi.org/10.1017/CBO9780511665707

Crittenden, P. M., \& DiLalla, D. L. (1988). Compulsive compliance: The development of an inhibitory coping strategy in infancy. Journal of
Abnormal Child Psychology, 16(5), 585-599. https://doi.org/10.1007/ BF00914268

Crone, E. A., Will, G. J., Overgaauw, S., \& Güroğlu, B. (2014). Social decision-making in childhood and adolescence. In P. A. M. van Lange, B. Rockenbach \& T. Yamagishi (Eds.), Social dilemmas. New perspectives on reward and punishment (pp. 161-181). New York, NY: Oxford University Press. https://doi.org/10.1093/acprof: oso/9780199300730.001.0001

Dawes, R. M. (1980). Social dilemmas. Annual Review of Psychology, 31(1), 169-193. https://doi.org/10.1146/annurev.ps.31.020180.001125

Diamantopoulos, A., \& Winklhofer, H.M. (2001). Index construction with formative indicators: An alternative to scale development. Journal of Marketing Research, 38(2), 269-277.

Dufwenberg, M., Gächter, S., \& Hennig-Schmidt, H. (2011). The framing of games and the psychology of play. Games and Economic Behavior, 73(2), 459-478. https://doi.org/10.1016/j.geb.2011.02.003

Egger, H. L., Pine, D. S., Nelson, E., Leibenluft, E., Ernst, M., Towbin, K. E., \& Angold, A. (2011). The NIMH Child Emotional Faces Picture Set (NIMH-ChEFS): A new set of children's facial emotion stimuli. International Journal of Methods in Psychiatric Research, 20(3), 145156. https://doi.org/10.1002/mpr.343

Evans, G. W., Li, D., \& Whipple, S. S. (2013). Cumulative risk and child development. Psychological Bulletin, 139(6), 1342-1396. https://doi. org/10.1037/a0031808

Fan, C.-P. (2000). Teaching children cooperation - An application of experimental game theory. Journal of Economic Behavior \& Organization, 41(3), 191-209. https://doi.org/10.1016/S0167-2681(99)00072-4

Goodman, R. (1997). The strengths and difficulties questionnaire: A research note. Journal of Child Psychology and Psychiatry, 38(5), 581586. https://doi.org/10.1111/j.1469-7610.1997.tb01545.x

Gradin, V. B., Pérez, A., Macfarlane, J. A., Cavin, I., Waiter, G., Tone, E. B., ... Steele, J. D. (2016). Neural correlates of social exchanges during the Prisoner's Dilemma game in depression. Psychological Medicine, 46(6), 1289-1300. https://doi.org/10.1017/S0033291715002834

Gummerum, M., Hanoch, Y., \& Keller, M. (2008). When child development meets economic game theory: An interdisciplinary approach to investigating social development. Human Development, 51(4), 235261. https://doi.org/10.1159/000151494

Harbaugh, W. T., \& Krause, K. (2000). Children's altruism in public good and dictator experiments. Economic Inquiry, 38(1), 95-109. https:// doi.org/10.1111/j.1465-7295.2000.tb00006.x

Hardin, G. (1968). The tragedy of the commons. Science, 162(3859), 1243-1248.

Hu, L.-T., \& Bentler, P. M. (1999). Cutoff criteria for fit indexes in covariance structure analysis: Conventional criteria versus new alternatives. Structural Equation Modeling - A Multidisciplinary Journal, 6(1), 1-55.

Jaffee, S. R. (2017). Child maltreatment and risk for psychopathology in childhood and adulthood. Annual Review of Clinical Psychology, 13, 525-551. https://doi.org/10.1146/annurev-clinpsy-032816-045005

Jöreskog, K. G. (1999). How large can a standardized coefficient be? Unpublished Technical Report. Available from: http://www.ssicentral. com/lisrel/techdocs/HowLargeCanaStandardizedCoefficientbe.pdf [last accessed 05 September 2017].

Kay, C., \& Green, J. (2013). Reactive attachment disorder following early maltreatment: Systematic evidence beyond the institution. Journal of Abnormal Child Psychology, 41(4), 571-581. https://doi.org/10.1007/ s10802-012-9705-9

Keil, J., Michel, A., Sticca, F., Leipold, K., Klein, A. M., Sierau, S., ... White, L. O. (2017). The Pizzagame: A virtual public goods game to assess cooperative behavior in children and adolescents. Behavior Research Methods, 49(4), 1432-1443. https://doi.org/10.3758/ s13428-016-0799-9

Kim, J., \& Cicchetti, D. (2010). Longitudinal pathways linking child maltreatment, emotion regulation, peer relations, and psychopathology. Journal of Child Psychology and Psychiatry, 51(6), 706-716. 
King-Casas, B., \& Chiu, P. H. (2012). Understanding interpersonal function in psychiatric illness through multiplayer economic games. Biological Psychiatry, 72(2), 119-125. https://doi.org/10.1016/j. biopsych.2012.03.033

Kraemer, H. C., Measelle, J. R., Ablow, J. C., Essex, M. J., Boyce, W. T., \& Kupfer, D. J. (2003). A new approach to integrating data from multiple informants in psychiatric assessment and research: Mixing and matching contexts and perspectives. American Journal of Psychiatry, 160(9), 1566-1577. https://doi.org/10.1176/appi.ajp.160.9.1566

Ledyard, J. О. (1995). Public goods. A survey of experimental research. In J. H. Kagel \& A. E. Roth (Eds.), The handbook of experimental economics (Chap. 2). Princeton, NJ: Princeton University Press.

Little, T. D. (2013). Longitudinal structural equation modeling. New York, NY: The Guilford Press.

Luce, R. D., \& Raiffa, H. (1957). Games and decisions: Introduction and critical surveys. New York, NY: Wiley.

MacCallum, R. C., \& Browne, M.W.(1993). The use of causal indicators in covariance structure models: Some practical issues. Psychological Bulletin, 114(3), 533-541. https://doi.org/10.1037/0033-2909.114.3.533

Manly, J. T., Kim, J. E., Rogosch, F. A., \& Cicchetti, D. (2001). Dimensions of child maltreatment and children's adjustment: Contributions of developmental timing and subtype. Development and Psychopathology, 13, 759-782.

Manly, J. T., Oshri, A., Lynch, M., Herzog, M., \& Wortel, S. (2013). Child neglect and the development of externalizing behavior problems: Associations with maternal drug dependence and neighborhood crime. Child Maltreatment, 18(1), 17-29. https://doi. org/10.1177/1077559512464119

McClure, E. B., Parrish, J. M., Nelson, E. E., Easter, J., Thorne, J. F., Rilling, J. K., ... Pine, D. S. (2007). Responses to conflict and cooperation in adolescents with anxiety and mood disorders. Journal of Abnormal Child Psychology, 35(4), 567-577. https://doi.org/10.1007/ s10802-007-9113-8

McClure-Tone, E. B., Nawa, N. E., Nelson, E. E., Detloff, A. M., Fromm, S. J., Pine, D. S., \& Ernst, M. (2011). Preliminary findings: Neural responses to feedback regarding betrayal and cooperation in adolescent anxiety disorders. Developmental Neuropsychology, 36(4), 453472. https://doi.org/10.1080/87565641.2010.549876

McLaughlin, K. A., Sheridan, M. A., \& Lambert, H. K. (2014). Childhood adversity and neural development: Deprivation and threat as distinct dimensions of early experience. Neuroscience \& Biobehavioral Reviews, 47, 578-591. https://doi.org/10.1016/j.neubiorev.2014.10.012

Mokros, A., Menner, B., Eisenbarth, H., Alpers, G. W., Lange, K. W., \& Osterheider, M. (2008). Diminished cooperativeness of psychopaths in a prisoner's dilemma game yields higher rewards. Journal of Abnormal Psychology, 117(2), 406-413. https://doi. org/10.1037/0021-843X.117.2.406

Montañés, R. F., de Lucas, T. M. T., \& Rodríguez, M. A. M. (2003). Antisocial personality disorder evaluation with the prisoner's dilemma. Actas Españolas de Psiquiatría, 31(6), 307-314.

Muthén, B., \& Muthén, L. (2013). Mplus statistical software version 7.11. Los Angeles, CA: Muthén \& Muthén.

O'Brien, R. M. (2007). A caution regarding rules of thumb for variance inflation factors. Quality \& Quantity, 41(5), 673-690. https://doi. org/10.1007/s11135-006-9018-6

Olson, M. (1965). Logic of collective action: Public goods and the theory of groups. Cambridge, MA: Cambridge University Press.

Olweus, D. (2001). Peer harassment: A critical analysis and some important issues. In J. Juvonen \& S. Graham (Eds.), Peer harassment in school. The plight of the vulnerable and victimized (pp. 3-20). New York, NY: Guilford Press. https://doi.org/10.1111/desc.12375

Padilla-Walker, L. M., Carlo, G., \& Nielson, M. G. (2015). Does helping keep teens protected? Longitudinal bidirectional relations between prosocial behavior and problem behavior. Child Development, 86(6), 1759-1772. https://doi.org/10.1111/cdev.12411
Petter, S., Straub, D., \& Rai, A. (2007). Specifying formative constructs in information systems research. MIS Quarterly, 31(4), 623-656. https:// doi.org/10.2307/25148814

Pitula, C. E., Wenner, J. A., Gunnar, M. R., \& Thomas, K. M. (2017). To trust or not to trust: Social decision-making in post-institutionalized, internationally adopted youth. Developmental Science, 20(3), e12375.

Pulcu, E., Thomas, E. J., Trotter, P. D., McFarquhar, M., Juhász, G., Sahakian, B. J., ... Elliott, R. (2015). Social-economical decision making in current and remitted major depression. Psychological Medicine, 45(6), 1301-1313. https://doi.org/10.1017/S0033291714002414

Rand, D. G., \& Nowak, M. A. (2013). Human cooperation. Trends in Cognitive Sciences, 17(8), 413-425. https://doi.org/10.1016/j. tics.2013.06.003

Robins, C. J., Bagby, R. M., Rector, N. A., Lynch, T. R., \& Kennedy, S. H. (1997). Sociotropy, autonomy, and patterns of symptoms in patients with major depression: A comparison of dimensional and categorical approaches. Cognitive Therapy and Research, 21(3), 285-300. https:// doi.org/10.1023/A:1021874415967

Rutter, M., Sonuga-Barke, E. J., Beckett, C., Castle, J., Kreppner, J., Kumsta, R., ... Gunnar, M. R. (2010). Deprivation-specific psychological patterns: Effects of institutional deprivation. Monographs of the Society for Research in Child Development, 75(1), 232-247.

Sally, D., \& Hill, E. (2006). The development of interpersonal strategy: Autism, theory-of-mind, cooperation and fairness. Journal of Economic Psychology, 27(1), 73-97. https://doi.org/10.1016/j. joep.2005.06.015

Sharp, C. (2012). The use of neuroeconomic games to examine social decision making in child and adolescent externalizing disorders. Current Directions in Psychological Science, 21(3), 183-188. https:// doi.org/10.1177/0963721412444726

Sharp, C., Ha, C., \& Fonagy, P. (2011). Get them before they get you: Trust, trustworthiness, and social cognition in boys with and without externalizing behavior problems. Development and Psychopathology, 23(2), 647-658. https://doi.org/10.1017/S0954579410000003

Sierau, S., Brand, T., Manly, J. T., Schlesier-Michel, A., Klein, A. M., Andreas, A., ... White, L. O. (2017). A multisource approach to assessing child maltreatment from records, caregivers, and children. Child Maltreatment, 22(1), 45-57. https://doi. org/10.1177/1077559516675724

Sloman, L., \& Taylor, P. (2016). Impact of child maltreatment on attachment and social rank systems: Introducing an integrated theory. Trauma, Violence, \& Abuse, 17(2), 172-185. https://doi. org/10.1177/1524838015584354

Sörbom, D. (1975). Detection of correlated errors in longitudinal data. British Journal of Mathematical \& Statistical Psychology, 28(2), 138151. https://doi.org/10.1111/j.2044-8317.1975.tb00558.x

Sorgi, K. M., \& van't Wout, M. (2016). The influence of cooperation and defection on social decision making in depression: A study of the iterated Prisoner's Dilemma Game. Psychiatry Research, 246, 512-519. https://doi.org/10.1016/j.psychres.2016.10.025

Surbey, M. K. (2011). Adaptive significance of low levels of self-deception and cooperation in depression. Evolution and Human Behavior, 32(1), 29-40. https://doi.org/10.1016/j.evolhumbehav.2010.08.009

Teicher, M. H., \& Samson, J. A. (2013). Childhood maltreatment and psychopathology: A case for ecophenotypic variants as clinically and neurobiologically distinct subtypes. American Journal of Psychiatry, 170(10), 1114-1133. https://doi.org/10.1176/appi. ajp.2013.12070957

Thompson, R. A. (1990). Vulnerability in research: A developmental perspective on research risk. Child Development, 61(1), 1-16. https://doi. org/10.2307/1131043

Tomasello, M. (2014). A natural history of human thinking. Cambridge, MA: Harvard University Press.

Vachon, D. D., Krueger, R. F., Rogosch, F. A., \& Cicchetti, D. (2015). Assessment of the harmful psychiatric and behavioral effects of 
different forms of child maltreatment. JAMA Psychiatry, 72(11), 11351142. https://doi.org/10.1001/jamapsychiatry.2015.1792

van Hoorn, J., van Dijk, E., Meuwese, R., Rieffe, C., \& Crone, E. A. (2016). Peer influence on prosocial behavior in adolescence. Journal of Research on Adolescence, 26(1), 90-100. https://doi.org/10.1111/ jora.12173

van Lange, P. A. M., Joireman, J., Parks, C. D., \& van Dijk, E. (2013). The psychology of social dilemmas: A review. Organizational Behavior and Human Decision Processes, 120(2), 125-141. https://doi. org/10.1016/j.obhdp.2012.11.003

van Lange, P., Klapwijk, A., \& van Munster, L. M. (2011). How the shadow of the future might promote cooperation. Group Processes \& Intergroup Relations, 14(6), 857-870. https://doi. org/10.1177/1368430211402102

Vogelsang, M., Jensen, K., Kirschner, S., Tennie, C., \& Tomasello, M. (2014). Preschoolers are sensitive to free riding in a public goods game. Frontiers in Psychology, 5(729), 1-9.

White, L. O., Ising, M., von Klitzing, K., Sierau, S., Michel, A., Klein, A. M., ... Stalder, T. (2017). Reduced hair cortisol after maltreatment mediates externalizing symptoms in middle childhood and adolescence. Journal of Child Psychology and Psychiatry, 58(9), 998-1007. https:// doi.org/10.1111/jcpp.12700

White, L. O., Klein, A. M., Kirschbaum, C., Kurz-Adam, M., Uhr, M., Müller-Myhsok, B., ... von Klitzing, K. (2015). Analyzing pathways from childhood maltreatment to internalizing symptoms and disorders in children and adolescents (AMIS): A study protocol. BMC Psychiatry, 15(126), 1-18.
Zahn-Waxler, C., \& van Hulle, C. (2011). Empathy, guilt, and depression: When caring for others becomes costly to children. In B. Oakley, A. Knafo, G. Madhavan \& D. S. Wilson (Eds.), Pathological altruism (pp. 321-345). New York, NY: Oxford University Press.

Zeanah, C. H., \& Gleason, M. M. (2015). Annual research review: Attachment disorders in early childhood-clinical presentation, causes, correlates, and treatment. Journal of Child Psychology and Psychiatry, 56(3), 207-222. https://doi.org/10.1111/jcpp.12347

Zelmer, J. (2003). Linear public goods experiments: A metaanalysis. Experimental Economics, 6, 299-310. https://doi. org/10.1023/A:1026277420119 